\title{
Granuloma piogênico oral: relato de caso clínico
}

\author{
Oral pyogenic granuloma: climical case report \\ Granuloma piógeno oral: reporte de caso clínico
}

Recebido: 25/11/2021 | Revisado: 04/12/2021 | Aceito: 11/12/2021 | Publicado: 19/12/2021

\author{
Maira Janiely Porto Gomes \\ ORCID: https://orcid.org/0000-0001-8797-5481 \\ Centro Universitário Mário Pontes Jucá, Brasil \\ E-mail: maira.janiely@hotmail.com \\ Maria Aparecida dos Santos Satirio \\ ORCID: https://orcid.org/0000-0002-0137-2477 \\ Centro Universitário Mário Pontes Jucá, Brasil \\ E-mail: cidasatirio@gmail.com \\ Melka Coelho Sá \\ ORCID: https://orcid.org/0000-0002-6712-4601 \\ Universidade Federal de Sergipe, Brasil \\ E-mail: melkasa@yahoo.com.br \\ Luiz Arthur Barbosa da Silva \\ ORCID: https://orcid.org/0000-0003-0031-4950 \\ Centro Universitário Mário Pontes Jucá, Brasil \\ E-mail: larthurbarbosa@hotmail.com
}

\begin{abstract}
Resumo
O granuloma piogênico é uma lesão benigna relativamente comum que pode acometer pacientes de todas as idades, sendo mais prevalente em adultos jovens e com predileção pelo sexo feminino, principalmente, no período gestacional. Sua etiologia não é totalmente compreendida, mas parece ter natureza reacional. Normalmente, apresenta-se como um nódulo de crescimento exofítico, coloração, tipicamente, avermelhada, superfície ulcerada, facilmente sangrante. A lesão é tratada convencionalmente através de remoção cirúrgica, sendo essencial a eliminação dos fatores irritativos locais para redução da ocorrência de recidivas. O presente trabalho tem como objetivo relatar um caso de Granuloma piogênico diagnosticado em uma paciente do sexo feminino, 18 anos de idade, que apresentou lesão localizada em papila interdental, entre dentes 12 e 13, com evolução assintomática de, aproximadamente, 6 meses. A lesão foi tratada cirurgicamente e o material removido foi encaminhado ao laboratório de Anatomia patológica para confirmação do diagnóstico. Após 30 dias de acompanhamento pós-operatório, observou-se satisfatório reparo tecidual e a paciente encontra-se há 1 ano sem sinais de recidiva da lesão.
\end{abstract}

Palavras-chave: Granuloma piogênico; Diagnóstico; Tratamento.

\begin{abstract}
Pyogenic granuloma is a relatively common benign lesion that affects patients of all ages, being more prevalent in young adults and with a predilection for females, especially during pregnancy. Its etiology is not fully understood, but it appears to be reactional in nature. It is sually presents as an exophytic growth nodule, typically reddish in color, displaying an ulcerated surface that bleeds easily. The lesion is conventionally treated through surgical removal, and the removal of local irritant factors is paramount to reduce recurrences. This paper aims to report a Pyogenic Granuloma case diagnosed in an 18-year-old female patient who presented a lesion located in the interdental papilla, between teeth 12 and 13, with an asymptomatic evolution of about 6 months. The lesion was surgically treated and the removed material was sent to a pathological anatomy laboratory for diagnosis confirmation. Satisfactory tissue repair was observed after 30 days of postoperative follow-up, and the patient exhibited no signs of lesion recurrence for 1 year.
\end{abstract}

Keywords: Pyogenic granuloma; Diagnosis; Treatment.

\section{Resumen}

El granuloma piógeno es una lesión benigna relativamente común que puede afectar a pacientes de todas las edades, siendo más prevalente en adultos jóvenes y con predilección por el sexo femenino, principalmente durante el embarazo. No se comprende completamente su etiología, pero parece ser de naturaleza reactiva. Suele presentarse como un nódulo de crecimiento exofítico de color típicamente eritematoso, con superficie ulcerada que sangra con facilidad. La lesión se trata de forma convencional mediante remoción quirúrgica, siendo esencial la eliminación de los factores irritantes locales para reducir las recurrencias. El presente trabajo tiene como objetivo reportar un caso de granuloma piógeno diagnosticado en una paciente de sexo femenino de 18 años que presentó una lesión localizada en la papila interdental, entre los dientes 12 y 13, con una evolución asintomática de aproximadamente 6 meses. La lesión fue tratada quirúrgicamente y el material removido se envió al laboratorio de anatomía patológica para 
confirmar el diagnóstico. Después de 30 días de seguimiento postoperatorio, se observó una reparación tisular satisfactoria y la paciente no presenta signos de recurrencia de la lesión desde hace 1 año.

Palabras clave: Granuloma piógeno; Diagnóstico; Tratamiento.

\section{Introdução}

O granuloma piogênico é, tradicionalmente, considerado uma lesão benigna não-neoplásica de natureza provavelmente reacional, correspondendo a aproximadamente 26,8 - 32\% de todas as lesões reativas dos maxilares (IsazaGuzma et al., 2012; Marla, et. al. 2016).

Esta patologia pode acometer pacientes de qualquer idade e ambos os sexos, embora seja mais comum em adultos jovens, com predileção pelo sexo feminino, provavelmente, em virtude da influência hormonal sobre a proliferação vascular. Este motivo explica também o aumento da sua incidência em mulheres durante o período gestacional (Jané-Salas et al., 2015).

Clinicamente, pode ser observado um aumento de volume exofítico, com superfície lisa ou lobulada, implantação séssil ou pedunculada, com coloração, tipicamente, avermelhada e apresentando ocasionais ulcerações. Comumente, é indolor e exibe sangramento espontâneo em virtude da sua alta vascularização (Bugshan et al., 2015; Sachdeva, 2015). O tamanho pode variar de poucos milímetros até alguns centímetros, raramente excedendo os $25 \mathrm{~mm}$ (Martins-Filho et al., 2011). Em aproximadamente 75\% dos casos, a gengiva é o sítio acometido (Asnaashari, et al., 2014). Radiograficamente, não apresenta alterações, apesar de em raros casos ser evidenciado rarefação óssea alveolar localizada. (Kamal et al., 2012; Ka et al., 2013).

$\mathrm{Na}$ maioria dos casos, o granuloma piogênico é parcial ou completamente revestido por epitélio pavimentoso estratificado. As ulcerações na superfície podem estar presentes, sendo recobertas por membrana fibrinopurulenta. A maior parte da lesão é formada por uma massa lobulada ou não lobulada de reação de granulação. A presença de inúmeros espaços vasculares e a proliferação de células endoteliais são aspectos característicos. A quantidade de fibras colágenas no tecido conjuntivo é variável. O infiltrado inflamatório é, predominantemente, misto, composto por neutrófilos, plasmócitos e linfócitos. Além disso, observa-se que lesões antigas, podem exibir áreas de fibrose (Marla et al., 2016; Demirkan, 2017; Poudel et al., 2018).

O tratamento consiste, principalmente, em remoção cirúrgica associada à eliminação dos fatores irritantes, como, por exemplo, cálculo dentário, restaurações insatisfatórias e raízes dentárias residuais (Ka, et al., 2013; Jané-Salas et al., 2015).

Desta forma, o objetivo do presente estudo é relatar um caso de granuloma piogênico bem como comparar os aspectos observados com os achados presentes na literatura científica especializada.

\section{Metodologia}

Trata-se de um relato descritivo do caso clínico de uma única paciente, com ênfase nas características clínicas, métodos de diagnóstico e tratamento da patologia estudada. A paciente assinou o Termo de Consentimento Livre e Esclarecido (TCLE) e se mostrou de acordo com a utilização das imagens (sem sua identificação) para enriquecimento da comunidade científica.

\section{Relato de Caso}

Paciente do sexo feminino, 18 anos, melanoderma, compareceu ao consultório odontológico apresentando lesão exofítica, nodular, pediculada, com coloração avermelhada, superfície com áreas ulceradas, medindo aproximadamente $1,5 \mathrm{~cm}$, localizada na região da papila interdental, entre dentes 13 e 12, com evolução de aproximadamente 6 meses (Figura 1). Paciente apresentava acúmulo de biofilme e presença de cálculo dentário. Diante das características observadas, foram levantadas as hipóteses diagnósticas de granuloma piogênico e lesão periférica de células gigantes. 
Figura 1. Aspecto clínico. Lesão nodular, coloração avermelhada na papila interdental entre dentes 13 e 12.

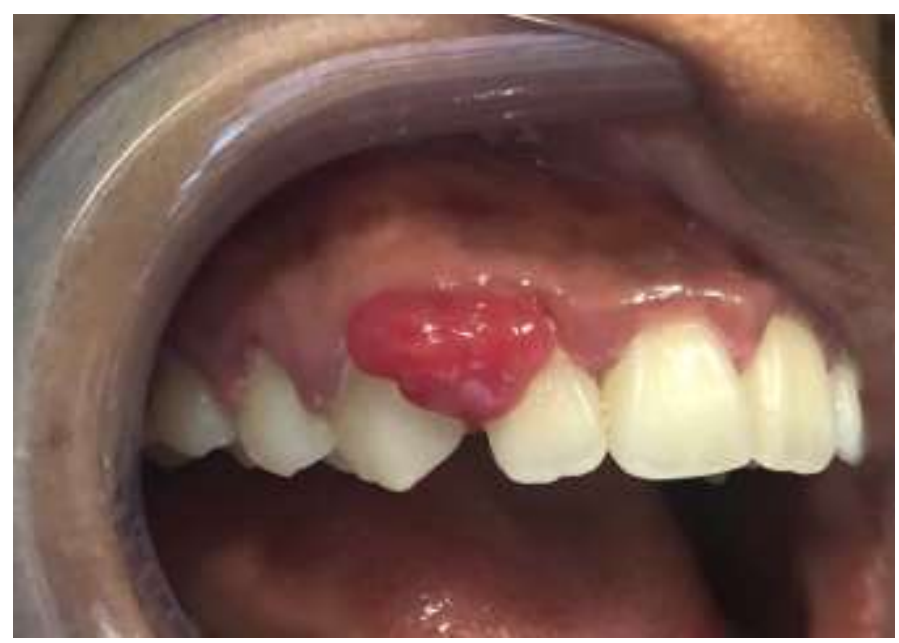

Fonte: Autores (2021).

A lesão foi submetida à biópsia excisional, sob anestesia local (Figura 2A), sem intercorrências. Após a remoção, os dentes da região foram raspados para eliminação dos cálculos dentários com o objetivo de eliminar fatores irritativos locais associados ao seu desenvolvimento (Figura 2B). Na região da ferida cirúrgica, realizou-se sutura simples (Figura 2C). O material removido (Figura 2D) foi fixado em formol a $10 \%$ e em seguida encaminhado ao laboratório de anatomia patológica para confirmação diagnóstica por meio de análise histopatológica.

Figura 2. Biópsia excisional. A. Anestesia local. B. Raspagem de cálculos dentários dos dentes da região da lesão. C. Sutura D. Material removido.

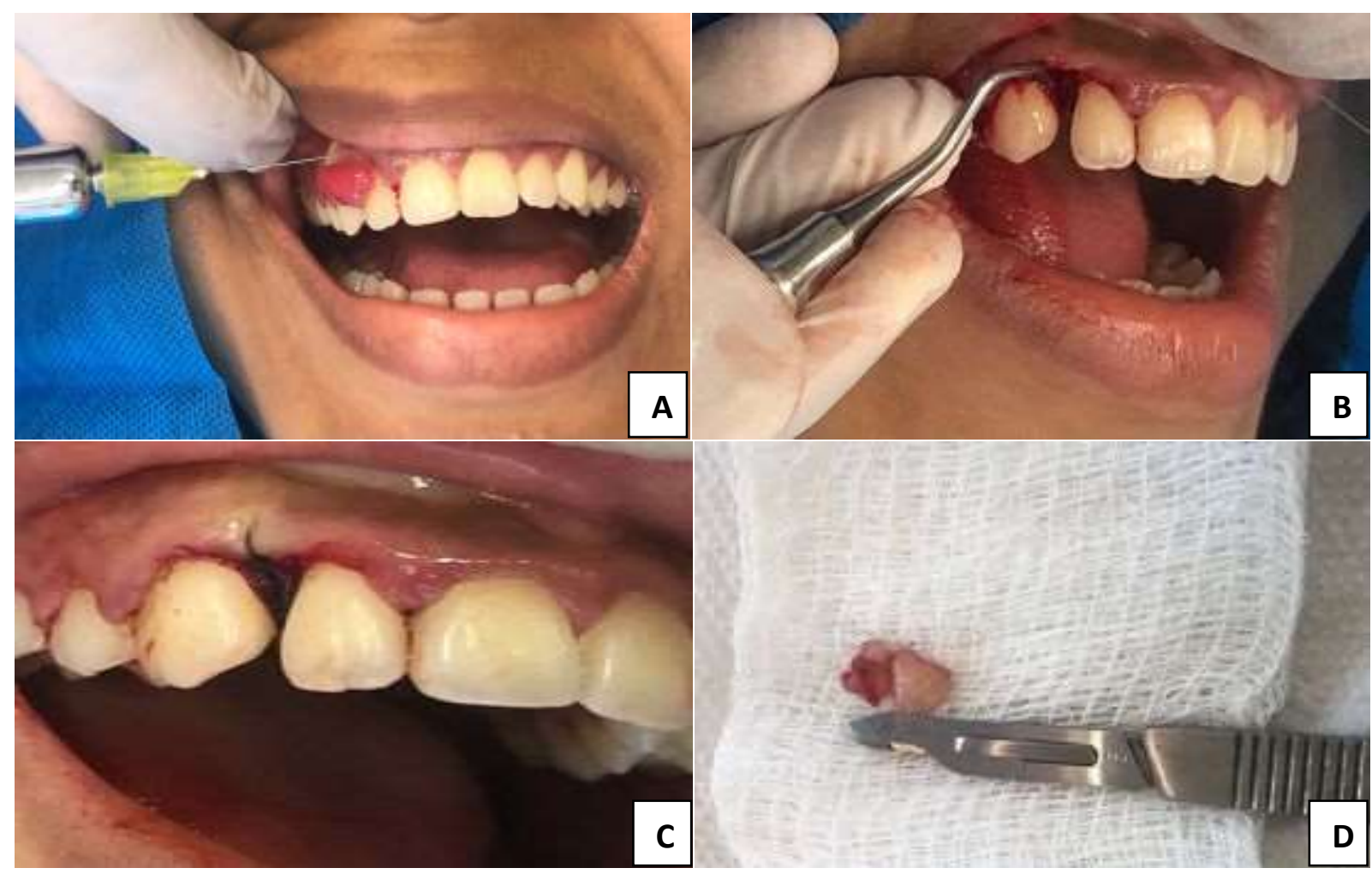

Fonte: Autores (2021). 
Research, Society and Development, v. 10, n. 16, e589101623876, 2021

(CC BY 4.0) | ISSN 2525-3409 | DOI: http://dx.doi.org/10.33448/rsd-v10i16.23876

Os cortes histopatológicos corados em hematoxilina-eosina e analisados sob microscopia de luz revelaram fragmento de mucosa oral com superfície ulcerada, recoberta por membrana fibrionopurulenta. No tecido conjuntivo subjacente, nota-se presença de reação de granulação, com moderado a intenso infiltrado inflamatório, proliferação de células endoteliais e espaços vasculares de tamanhos variados, sendo, portanto, confirmado o diagnóstico de granuloma piogênico (Figura 3 ).

Figura 3. Aspecto histopatológico. Área de ulceração recoberta por membrana fibrinopurulenta, com reação de granulação exibindo infiltrado inflamatório, células endoteliais e espaços vasculares.

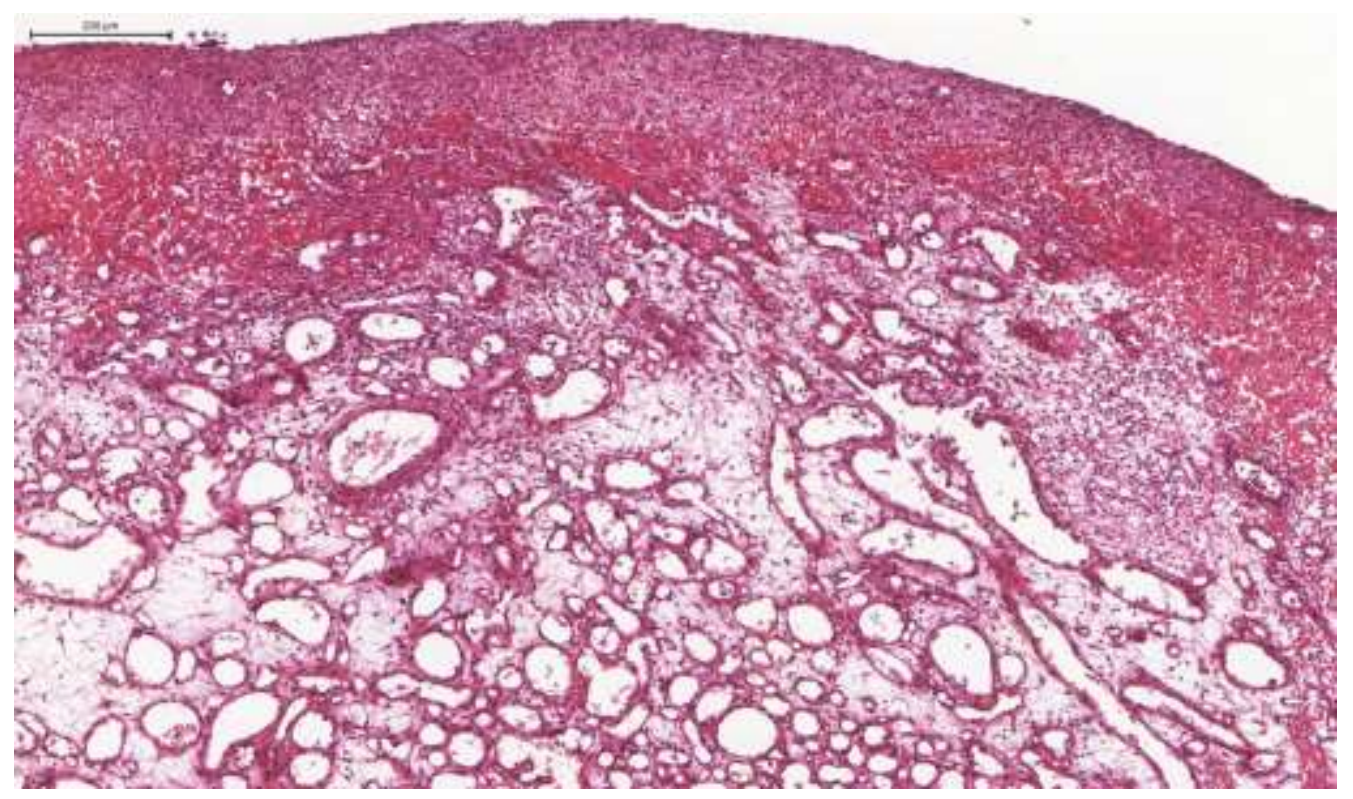

Fonte: Autores (2021).

A paciente retornou após 7 dias para remoção da sutura (Figura 4A) e após 30 dias para controle pós-operatório tendo sido observado reparo tecidual satisfatório (Figura 4B). A mesma foi orientada a buscar atendimento odontológico para completar a raspagem e alisamento radicular e também em relação à importância de manter uma boa higienização bucal. A paciente encontra-se há um ano sem sinais de recidivas da lesão.

Figura 4. A e B - Sete dias após 7 dias para remoção da sutura.

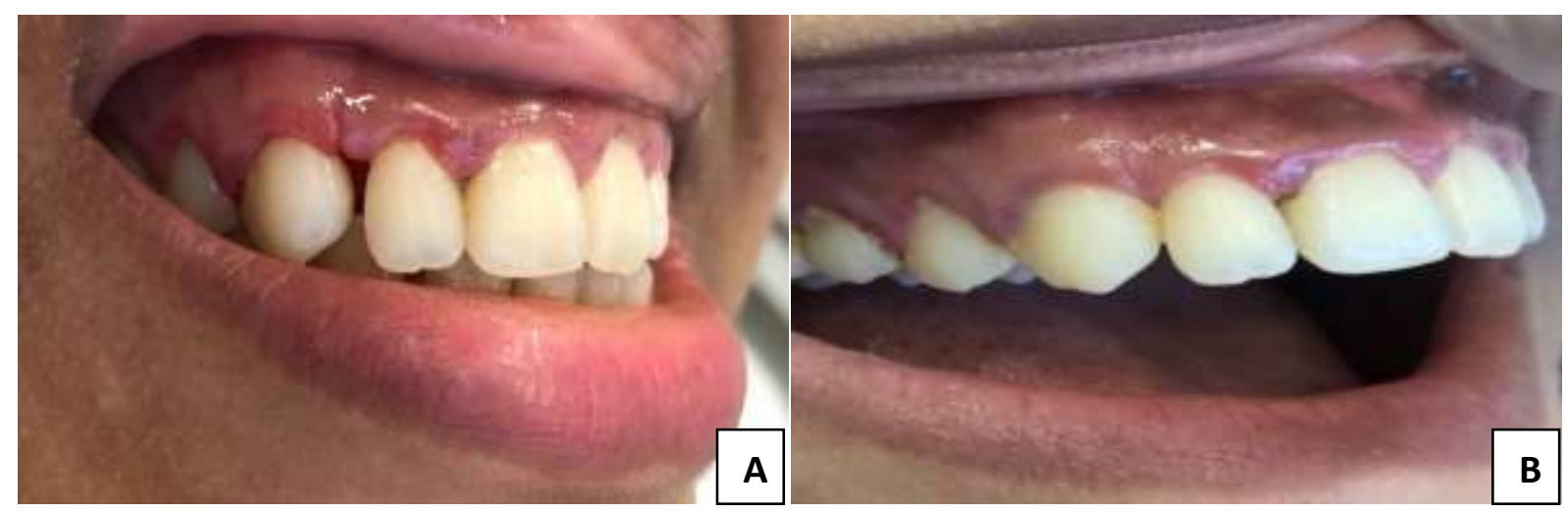

Fonte: Autores (2021). 


\section{Discussão}

O granuloma piogênico é uma patologia relativamente comum cuja nomenclatura não condiz com os seus aspectos etiopatológicos. Apesar de originalmente acreditar-se que era causado por micro-organismos piogênicos, sabe-se que a lesão não está relacionada a processos infecciosos. Assim, o termo "granuloma piogênico" é impróprio, apear de amplamente utilizado (Kamal et al., 2012; Bugshan et al., 2015; Sachdeva, 2015).

Embora a sua etiopatogenia não esteja bem estabelecida, vários fatores podem influenciar no desenvolvimento desta lesão, tais como: irritação crônica, higiene oral deficiente e os hormônios sexuais femininos (Seyedmajidi et al., 2015; Poudel et al., 2018). A paciente do caso em questão exibia deficiente higienização oral, tendo sido observado acúmulo de biofilme e presença de cálculo dentário na região de aparecimento da lesão. Vários estudos têm demonstrado uma maior associação do surgimento de granulomas piogênicos em indivíduos do sexo feminino. Esta associação vem sendo atribuída às alterações endócrinas e imunológicas significativas que as mulheres apresentam, aumentando, assim, a vulnerabilidade para desordens gengivais e infecciosas, principalmente durante o período gestacional, em que a frequência de acometimento é comumente de 0,5-5\% (Kruger et al,. 2013). Durante a anamnese, nossa paciente negou estar em período gestacional.

O granuloma piogênico na cavidade oral acomete, com maior frequência, a gengiva, mas também pode ser observada em outras regiões como: lábio, língua e mucosa jugal. Ao exame físico, apresenta-se como um nódulo exofítico, de crescimento relativamente rápido, com coloração avermelhada, que pode exibir sangramento espontâneo devido a sua alta vascularização (Mendonça et al,. 2010; Menezes et al., 2014; Jané-salas., 2015; Marla et al., 2016). Vale ressaltar que a lesão, normalmente, não apresenta sintomatologia dolorosa, porém pode provocar desconforto estético, sangramento e problemas funcionais relacionados à mastigação, deglutição e fala. (Diniz et al, 2021). As características clínicas apresentadas no presente caso corroboram os aspectos descritos na literatura.

Nos exames de imagem, o granuloma piogênico não exibe aspecto que o defina, tendo em vista que a lesão é restrita a tecido mole, porém, estes exames podem ser solicitados para ajudar na exclusão de diagnósticos de outras patologias (Parikh, et al., 2011). Neste presente relato, exames de imagem não foram solicitados.

No que diz respeito aos diagnósticos diferenciais do granuloma piogênico, podem ser consideradas entidades patológicas como lesão periférica de células gigantes, fibroma ossificante periférico e malformação vascular, sendo, portanto, essencial a realização de exame histopatológico para determinação do diagnóstico definitivo (Asnaashari, et al., 2014; Asnaashari, et al., 2015).

Na literatura, cita-se a possibilidade de regressão espontânea do granuloma piogênico, mas na maioria dos casos, como no relato aqui apresentado, há a necessidade de abordagem terapêutica da lesão, através de modalidades como remoção cirúrgica convencional (principal forma de tratamento), crioterapia ou cirurgia a laser (Gonçales, et al., 2010; Asnaashari, et al., 2015; Koo et al., 2017). É importante considerar que a intervenção utilizada priorize a redução do risco de recidiva e um menor comprometimento estético. Destaca-se, também, que para esta redução do risco de recidiva, é essencial a eliminação dos fatores irritativos locais associados ao surgimento da lesão (Diniz et al, 2021). Após a remoção do nódulo na gengiva da paciente aqui descrita, realizou-se raspagem periodontal para eliminação de cálculos dentários adjacentes à lesão.

\section{Considerações Finais}

Por sua relativa frequência na população, destaca-se a necessidade do Cirurgião-Dentista reconhecer as características do granuloma piogênico para encaminhar ou tratar de forma adequada os pacientes acometidos. Destaca-se também a necessidade da remoção de fatores irritativos locais associados ao desenvolvimento da doença. Com a realização de mais estudos, espera-se melhor compreender o processo de etiopatologia do granuloma piogênico. A paciente aqui apresentada encontra-se há um ano sem sinais de recidiva da lesão. 
Research, Society and Development, v. 10, n. 16, e589101623876, 2021

(CC BY 4.0) | ISSN 2525-3409 | DOI: http://dx.doi.org/10.33448/rsd-v10i16.23876

\section{Referências}

Asnaashari, M. et al.(2014) Posthaste Outgrow of lip Pyogenic Granuloma after Diode Laser removal. Journal of Lasers in Medical Sciences. 5(2), 92-95, 2014 .

Asnaashari, M. et al. (2015). Expedited removal of pyogenic granuloma by diode laser in a pediatric patient. Journal of Lasers in Medical Sciences, 6(1):40-4.

Bugshan, A., Patel, H., Garber, K., \& Meiller, T. F. (2015). Alternative therapeutic approach in the treatment of oral pyogenic granuloma. Case reports in oncology, 8(3), 493-497.

Demirkan S. (2017) Management of a Recurrent Pyogenic Granuloma of the Inferior Lip with Pulsed Dye Laser: A Case Report. J Am Coll Clin Wound Spec, 8(1-3):39-41.

Diniz, D. A., Cunha, J. S., Mendonça, T. L., Nascimento, V. H., Silva, C. C., Gonçalves, K. K., \& Maciel, F. A. (2021). Granuloma piogênico atípico: diagnóstico e tratamento cirúrgico. RSBO, 18(1), 173-179.

Gonçales, E. S. et al. (2010). Pyogenic granuloma on the upper lip: na unusual location. Journal Of Applied Oral Science. 18(5), 538-541, 2010.

Isaza-Guzmán, D. M., Teller-Carrero, C. B., Laberry-Bermúdez, M. P., González-Pérez, L. V., \& Tobón-Arroyave, S. I. (2012). Assessment of clinicopathological characteristics and immunoexpression of COX-2 and IL-10 in oral pyogenic granuloma. Archives of oral biology, 57(5), 503-512.

Jané-Salas, E., Albuquerque, R., Font-Muñoz, A., González-Navarro, B., Estrugo Devesa, A., \& López-López, J. (2015). Pyogenic granuloma/peripheral giant-cellgranuloma associated with implants. International journal of dentistry, 2015.

Kamala, K. A., et al. (2013) Pyogenic granuloma on the upper labial mucosa: a case report. Journal of clinical and diagnostic research: JCDR, 7(6), 1244-6.

Kamal, R.; Dahiya, P., \& Puri, Abhiney. (2012) Oral pyogenic granuloma: Various concepts of etiopathogenesis. J Oral Maxillofac Pathol. 16(1), 79-82.

Koo, M. G. Lee, S. H.; Han, S. E. (2017). Pyogenic Granuloma: A Retrospective Analysis of Cases Treated Over a 10-Year. Archives Of Craniofacial Surgery. 18(1), 16-20.

Krüger, M. S. D. M., Rosa, D. P. D., Pappen, F. G., Romano, A. R., \& Corrêa, F. D. O. B. (2013). Granuloma gravídico-relato de caso. Odontologia ClínicoCientífica (Online), 12(4), 293-295.

Marla, V., Shrestha, A., Goel, K., \& Shrestha, S. (2016). The histopathological spectrum of pyogenic granuloma: a case series. Case reports in dentistry, 2016.

Martins-Filho, P. R. S., Souto, M. L. S., Piva, M. R., \& Takeshita, W. M. (2014). Lesões maxilofaciais: um levantamento de 762 casos da Universidade Federal de Sergipe, Brasil. Rev. odontol. UNESP, 43(3), 185-190.

Maryam Seyedmajidi, S. S., Hashemipour, G., Bijani, A., \& Ehsani, H. (2015). Immunohistochemical evaluation of angiogenesis related markers in pyogenic granuloma of gingiva. Asian Pacific Journal of Cancer Prevention, 16(17), 7513-7516.

Mendonça, J. C. G., Gaetti-Jardim, E. C., Macena, J. A., Teixeira, F. R., dos Santos, C. M., Oliveira, M. M., Mosocatto, D. C., \& de Quadros, D. C. (2015). Granuloma piogênico de grandes proporções: relato de caso clínico-cirúrgico. Archives of Health Investigation, 4(3).

Menezes, R. E. R. D., Silva, S. O. D., Zanata, A., De Carli, B. M. G., Dutra, M. D. Z., \& De Carli, J. P. (2014). Patogênese e aspectos clínicos do granuloma gravídico: Relato de caso e revisão de literatura. Salusvita, 33(1), 111-127.

Parikh, K. et al. (2011). Pyogenic Granuloma: A report of three cases. Journal Dentistry Sciences. Georgia, 4(2), $194-196$.

Poudel, P. Chaurasia, N. Marla, V. Srii, R. (2018). Pyogenic granuloma of the upper lip: A common lesion in an uncommon location. J Taibah Univ Med Sci, 14(1):95-98.

Sachdeva, S. K. (2015) Extragingival Pyogenic Granuloma: an Unusual Clinical Presentation. J Dent (Shiraz), 16(3 Suppl):282-285. 